\title{
Nainital Microlensing Survey - detection of short period Cepheids in the disk of M 31
}

\author{
Y. C. Joshi ${ }^{1,2}$, D. Narasimha ${ }^{3}$, A. K. Pandey ${ }^{1}$, R. Sagar ${ }^{1}$ \\ 1 Aryabhatta Research Institute of Observational Sciences (ARIES), Manora peak, Nainital, India \\ e-mail: yogesh@aries.res.in \\ 2 Astrophysics Research Centre, School of Mathematics \& Physics, Queen's University, Belfast, BT7 1NN, UK \\ 3 Tata Institute of Fundamental Research, Homi Bhabha Road, Mumbai, India
}

Received 6 October 2009 / Accepted 28 December 2009

ABSTRACT

\begin{abstract}
Context. Cepheids are the primary distance indicators for the external galaxies, so discovery of large number of Cepheid variables in far-off galaxies offers a unique opportunity to determine the accurate distance of the host galaxy through their period-luminosity relation.

Aims. The main purpose of this study is to identify short-period and relatively faint Cepheids in the crowded field of M 31 disk, which was observed as part of the Nainital Microlensing Survey.

Methods. The Cousins $R$ and $I$ band photometric observations were carried out in the direction of M 31 with the aim of detecting microlensing events. The data was obtained with a 1-m telescope on more than 150 nights over the period between November 1998 and January 2002. The data was analysed using the pixel technique and the mean magnitudes of the Cepheids were determined by correlating their pixel fluxes with the corresponding PSF-fitted photometric magnitudes.

Results. In the present study we report identification of short-period Cepheid variables in the M 31 disk. We present a catalogue of 39 short-period $(P<15$ days $)$ Cepheids in a $\sim 13^{\prime} \times 13^{\prime}$ region of the M 31 disk and give positions and pulsation periods along with their $R$ and $I$ bands photometric magnitudes wherever possible. Most of the Cepheids are found with $R$ (mean) 20-21 mag, and the dense phase coverage of our observations enabled us to identify Cepheids with periods as short as 3.4 days. The period distribution of these Cepheids peaks at $\log P \sim 0.9$ and 1.1 days.

Conclusions. We demonstrate that using the pixel method, faint and short-period Cepheids in M 31 can even be detected with smallsize telescopes and moderate observing conditions.
\end{abstract}

Key words. methods: observational - techniques: photometric - Cepheids

\section{Introduction}

In the past decade, the Andromeda galaxy (M31) has been a search target for gravitational microlensing events by several wide-field surveys e.g. AGAPE (Ansari et al. 1997, 1999), Columbia-VATT (Crotts \& Tomaney 1996), POINT-AGAPE (Auriére et al. 2001; Paulin-Henriksson et al. 2003), WeCAPP (Riffeser et al. 2001), MEGA (de Jong et al. 2004), and Angstrom (Kerins et al. 2006). To distinguish microlensing events from known types of variable stars, these surveys need continuous observations for a long time span with good temporal sampling though for a short-period of time each night. Such observations are therefore perfectly suited to the detection of variable stars (e.g. Cepheids, Miras) and optical transient events. Several groups dedicated to searching for microlensing events in the direction of M 31 have already uncovered a large number of variable stars, most of which are previously unidentified (Joshi et al. 2003; Ansari et al. 2004; An et al. 2004; Fliri et al. 2006), as well as nova outbursts (Joshi et al. 2004, Darnley et al. 2004) as a major by-product.These variable stars are of cosmological interest, particularly Cepheids, which are massive $\left(M \sim 3-20 M_{\odot}\right)$ pulsating stars placed in the instability strip of the Hertzsprung-Russell diagram. They can be identified by their characteristic "saw-tooth" shaped light curves and high intrinsic brightness. The correlation of the period of pulsation with their intrinsic magnitudes makes them useful for measuring distances to galaxies in the Local Group and nearby clusters of galaxies. In recent times, substantial work has been done to determine the distance of M 31 using population I Cepheids through their period-luminosity relation, with a broad range of distances (730 to $790 \mathrm{kpc}$ ) reported by different groups (e.g. Freedman et al. 2001; Joshi et al. 2003; Vilardell et al. 2007).

Starting in November 1998, we undertook a long-term project, the "Nainital Microlensing Survey" to search for microlensing events towards M31 using a 1-m telescope in Nainital, India. The survey has had good temporal coverage during September/October to January for four consecutive observing seasons of M31, and it offers an excellent opportunity to search for variable stars and optical transients in the disk of M31. In our earlier survey papers, we reported variable stars including long-period Cepheids and irregular variables (Joshi et al. 2003, hereafter referred as JOS03), classical novae (Joshi et al. 2004), and a microlensing candidate event (Joshi et al. 2005). In this paper we present a catalogue of short-period Cepheids $(P<15$ days $)$ detected in the survey. While a detailed description of our observations and reduction can be found in JOS03, a brief overview is given in Sect. 2. In Sect. 3 we describe the pixel analysis procedure used to identify Cepheids in our data. The detection procedure of Cepheids and their catalogue are given in Sects. 4 and 5 respectively. Our results are discussed in Sect. 6. 


\section{Observations and data reduction}

Cousins $R$ and $I$ band photometric observations of the target field centred at $\alpha_{2000}=0^{\mathrm{h}} 43^{\mathrm{m}} 38^{\mathrm{s}} ; \delta_{2000}=+41^{\circ} 09^{\prime}$. 1 , were obtained with a 1-m Sampurnanand Telescope at Manora Peak, Nainital, India. The total integration time during our survey ranges from $\sim 30$ min to $2 \mathrm{~h}$ each night, and a median seeing during the observations was $\sim 2.2$ arcsec. Because of time constraints, it was not possible to observe the target field in both the filters each night so we put an observing priority on the $R$ band. Images observed in poorer seeing than 3.5 arcsec were removed from our analysis to avoid blending problems since a large number of stars are present in the target field. In the 4 year long observing run, we were finally left with a total of 133 nights data in $R$ band and 115 nights data in $I$ band with a total time span of $\sim 1200$ days. In addition, we also observed the Landolt's standard field SA98 (Landolt 1992) on the photometric night of 25/26 October 2000 in order to derive the transformation equations to standard magnitudes. A log of observations in electronic form is given in JOS03.

The basic steps of image processing, which include bias subtraction, flat fielding, masking of bad pixels, and cosmic ray removal, were performed using IRAF ${ }^{1}$. To improve the signal-tonoise ratio, all images in a particular passband were combined on a nightly basis, resulting in a single image per filter per night.

Stellar photometry of all the images in both the filters were carried out for about 4400 resolved stars using DAOPHOT photometry (Stetson 1987). PSF was obtained for each frame using 25-30 relatively bright, uncontaminated stars. The DAOPHOT/ALLSTAR routine was used to calculate the instrumental magnitude of these stars. The absolute calibration was done using Landolt's (1992) standard field SA98. The typical photometric error was estimated to be about 0.04 mag for stars at $R=20 \mathrm{mag}$, increasing to $0.20 \mathrm{mag}$ at $R=22 \mathrm{mag}$.

\section{Image analysis using the pixel method}

Since our target field of M31 is largely composed of faint stars, all the stars are not well-resolved nor is each variable star sufficiently bright at minimum brightness to obtain reliable DAOPHOT photometry. The incompleteness in our data set begins at $R \sim 20$ and $I \sim 19.5$, which is precisely the brightness that we would expect of the lower luminosity short-period Cepheid variables. Therefore, in the present study, we used the pixel method to analyse our data, which relies on the monitoring of pixel light curves and their shape analysis. This method was originally proposed by Baillon et al. (1993) and implemented by Ansari et al. (1997) and others. In the pixel method, if a star of flux $F_{\text {star }}$ is increased, whether due to intrinsic variability or gravitationally lensed, then by subtracting the original flux from the amplified flux of the star, we see an increase in flux equal to $(A-1) F_{\text {star }}$ above the photon noise where $A$ is the flux amplification of the star. Thus by following $\Delta F$ with time, we in fact monitor the variation in the flux of the target star.

Our implementation of pixel method is described in detail in Joshi et al. (2005), where we report detecting the first microlensing candidate in our survey. To implement the pixel method in our data, we first chose a reference frame taken in good photometric conditions with low sky background and relatively good

\footnotetext{
1 Image Reduction and Analysis Facility (IRAF) is distributed by the National Optical Astronomy Observatories, which are operated by the Association of Universities for Research in Astronomy, Inc., under cooperative agreement with the National Science Foundation.
}

seeing $\left(\sim 1^{\prime \prime}\right.$.5). Images were normalized with respect to the reference frame in the following three steps.

1. We geometrically aligned all the images with better than \pm 0.05 arcsec accuracy through rotation and shifting with respect to the reference frame.

2. The photometric conditions were different on different nights during our observing runs, which we corrected by normalizing all the images with respect to the median background of the reference frame.

3 . To further reduce the fluctuations due to the seeing problem, we constructed a superpixel of $7 \times 7$ pixels $(\sim 2.5 \times$ $2.5 \operatorname{arcsec}^{2}$ ), whose combined flux is given by

$$
\phi_{\text {superpixel }}(i, j)=\sum_{k=i-3}^{i+3} \sum_{l=i-3}^{i+3} \phi_{\text {pixel }}(k, l)
$$

where $\phi_{\text {pixel }}(i, j)$ is the pixel flux at any pixel coordinate $(i, j)$.

In our subsequent discussion, we use the term pixel for convenience when referring to the superpixel. After corrections, the photon counts in any pixel are expected to only exhibit a flux variation $(\Delta F)$ above the background level if any star or stars falling over the pixel show intrinsic brightness variations. It is worth mentioning here that, to detect any variation in the flux, this change must be significantly above the background level. In our analysis, we considered flux variation as significant if at least 3 consecutive points were above the $3 \sigma$ level in each observing season. It is important to point out that the pixel method is more sensitive to the detection of faint but large amplitude than to bright but low amplitude variables (An et al. 2004).

\section{Identification of Cepheid variables}

While a substantial number of M 31 Cepheids are reported in the long-period range ( $P \sim 7-60$ days), short-period Cepheids are not well-reported, as they are relatively faint and have variations of smaller amplitude. In this study, we used our data to search for short-period Cepheids ( $P<15$ days). To identify these variables, we first masked all the bright stars $(R<19.5 \mathrm{mag})$ in a 10 pixel radius $(\sim 2 \times F W H M)$ in all the frames. The remaining pixels, we searched for variability in their light curves, and we identified few thousands pixels in our target field that were further analysed for their periodic variations.

\subsection{Period determination}

Following JOS03, we used a modified version of the Press \& Rybici (1989) FORTRAN program based on the method of Horne \& Baliunas (1986) to determine the period of the variable stars. This method uses a series of sinusoidal signals to best match the time series, hence find the period. The time series is convolved with sinusoidal curves until the peak of the convolution is found. On average, we had an $R$ band image every 3 days (with wide gaps between observing seasons), so we started searching for variations with a minimum period of 3 days and with an increment of 0.01 days. As this data had already been used to find longer period Cepheid variables using DAOPHOT profile fitting techniques (see JOS03), we searched for periods up to 15 days.

\subsection{Selection criteria to identify Cepheids}

A systematic search for variable stars in the data was performed by determining the shape of the periodic variations in the selected pixels. The $R$ band images with their improved temporal 
coverage and photometric accuracy were used to characterise the period variations in terms of mean brightness, period, and amplitude of the Cepheids. The $I$ band data was not used at this stage. Measurements were flagged as bad when the error in the pixel flux was more than 400 ADU so was discarded from further analysis. We initially only used those pixels that follow these criteria:

1. After the bad pixels rejection, individual pixel flux measurements are available for at least $50 \%$ of the total nights.

2. The pixel flux shows a periodic variation with a period less than 15 days

3. The amplitude of any periodic flux variation is greater than 200 ADU in the $R$ band data.

We thus shortlisted 1177 pixels that passed these criteria. Using the period determined in the previous section, we derived the phase for each observation. Since there are large errors in the individual pixel fluxes, we binned the data in 20 bins of width 0.05 in phase. For those bins with measurements, the weighted mean pixel flux and error were determined. The binned light curves have a smaller scatter, allowing better visual identification of Cepheid-like variability. A total of 39 Cepheids with periods ranging from $\sim 3.5$ to 15 days were identified. The $I$ band data was then binned after discarding all those pixels that have flux errors larger than 600 ADU. We phased the $I$ band data using the same period as estimated through $R$ band and found the periodic variations in all the Cepheids but NMS-M31V05. However, we still consider NMS-M31V05 as a Cepheid variable as the shape of its $R$ band phase light curve looks convincing.

In Figs. 1, 2, we show the light curves of 39 detected Cepheids in $R$ and $I$ bands, respectively, in order of increasing period. The period of each Cepheid is given at the top of its pixel light curve. Given the lesser observations in $I$ band, the $I$ band light curves have larger uncertainties than their $R$ band counterparts, particularly in the extremely short-period regime, which contains faint stars and exhibit low-amplitude variability.

\subsection{Determination of mean magnitude}

To determine the mean magnitude of Cepheids, we first calculated phase-weighted mean flux as

$F_{\text {mean }}=0.5 \sum_{i=1}^{\mathrm{n}}\left(\phi_{i}+1-\phi_{i-1}\right) F_{\text {pixel }}$

where $n$ is the total number of observations, and $\phi_{i}$ the phase of $i$ th observation in order of increasing phase after folding the period. The equation requires non-existent entities $\phi_{0}$ and $\phi_{n+1}$, which is set identical to $\phi_{n}$ and $\phi_{1}$ respectively.

Unlike in other pixel surveys (e.g. An et al. 2004; Fliri et al. 2006) where pseudo-magnitudes of the variable stars were determined from their flux variations, we calculated the absolute magnitude of the Cepheids using their photometric magnitudes if available through DAOPHOT photometry. To do this, whenever we identified a Cepheid-like light curve in the pixel method, we forcefully ran IRAF DAOPHOT "FIND" routine around that pixel position in all the frames of both the filters. We did not consider a star identified if it is more than 3 pixels $(\sim 1$ arcsec) different from the given pixel coordinates as it could be a different star or may have been the result of a blending problem. We did not find all the stars in all the images because most of these Cepheids were faint enough to be close to our detection limit and partly because of observations held in different sky conditions over the 4 year period. In the next step, we carried out PSF photometry around these stars to estimate their precise photometric magnitude and did not use any selection criteria to accept the magnitude unlike in JOS03. We found more than 30 photometric measurements for most of the Cepheids but could not find reliable photometric magnitudes for 6 Cepheids in $R$ band and 15 in $I$ band.

For any Cepheid, we correlated photometric magnitudes with their corresponding pixel fluxes to determine the background flux, which varied with spatial positions because of the large background gradient in the M 31 disk. We note here that all the images used to determine the pixel fluxes are background corrected and, therefore, contain the same background level at the position of the Cepheids. We derived a linear relation between the PSF-fitted photometric magnitudes converted into photometric fluxes and that of the pixel fluxes. We kept the slope fixed for all the Cepheids and neglected the colour term in the transformation as the error in our $I$ band photometry was expected to be larger than that from the colour term itself. In correlating the two fluxes, we derived the background flux only when the two data were correlated to $>80 \%$. For example, Fig. 3 shows the correlation between PSF-fitted photometric fluxes and pixel fluxes for a Cepheid NMS-M31V2. This star was identified in only 41 nights on the DAOPHOT photometric identification, because the star was fainter than $21 \mathrm{mag}$ in the $R$ band, even at its maximum brightness. We used 28 nights after three iterations of one-sigma clipping to determine the background flux at the position of this Cepheid. In a similar way, we determined the background flux for each Cepheid at their pixel positions. Using the fixed slope and background flux for each Cepheid, we converted the phase-weighted mean pixel flux into the mean magnitude for all 33 Cepheids in $R$ and 24 Cepheids in the $I$ band. The standard deviations in our magnitudes could be as much as 0.20 mag in $R$ and 0.30 mag in the $I$ band. The main source of error in the magnitudes is dominated by the transformation from pixel flux to photometric magnitude because of the lack of precise photometry towards the fainter end. It should be mentioned that most of these Cepheids are observed close to the detection limit of our telescope, and some of them may not even be detected in their minimum brightness phase.

\subsection{Astrometry}

Astrometry was performed on one of the best images obtained on a photometric night with relatively good seeing $\left(\sim 1^{\prime \prime} .7\right)$ and lower sky background. To convert the pixel coordinates $(X, Y)$ into celestial coordinates $(\alpha, \delta)$, reference positions of 324 bright stars from the USNO catalogue (Monet et al. 2003) were used to find linear astrometric parameters. The pixel positions of the detected Cepheids were then converted to the J2000 celestial coordinates in the equatorial system using the IRAF tasks of ccmap and $c c$ tran. The coordinates match within $\sim 1.0$ arcsec with those given in the Magnier catalogue (Magnier et al. 1992) and should be considered as the typical inaccuracy of our astrometry.

\section{The catalogue}

Using the pixel method as a detection technique for the faint Cepheids, we significantly increased the number of Cepheids in our target field of M31. A list of 39 short-period Cepheids identified in our survey is given in Table 1. The Cepheids are sorted in order of increasing period. The table contains the object identifier, right ascension (RA), declination (Dec), period $(P), R$ and $I$ band phase-weighted mean magnitudes $(\bar{R}, \bar{I})$, 


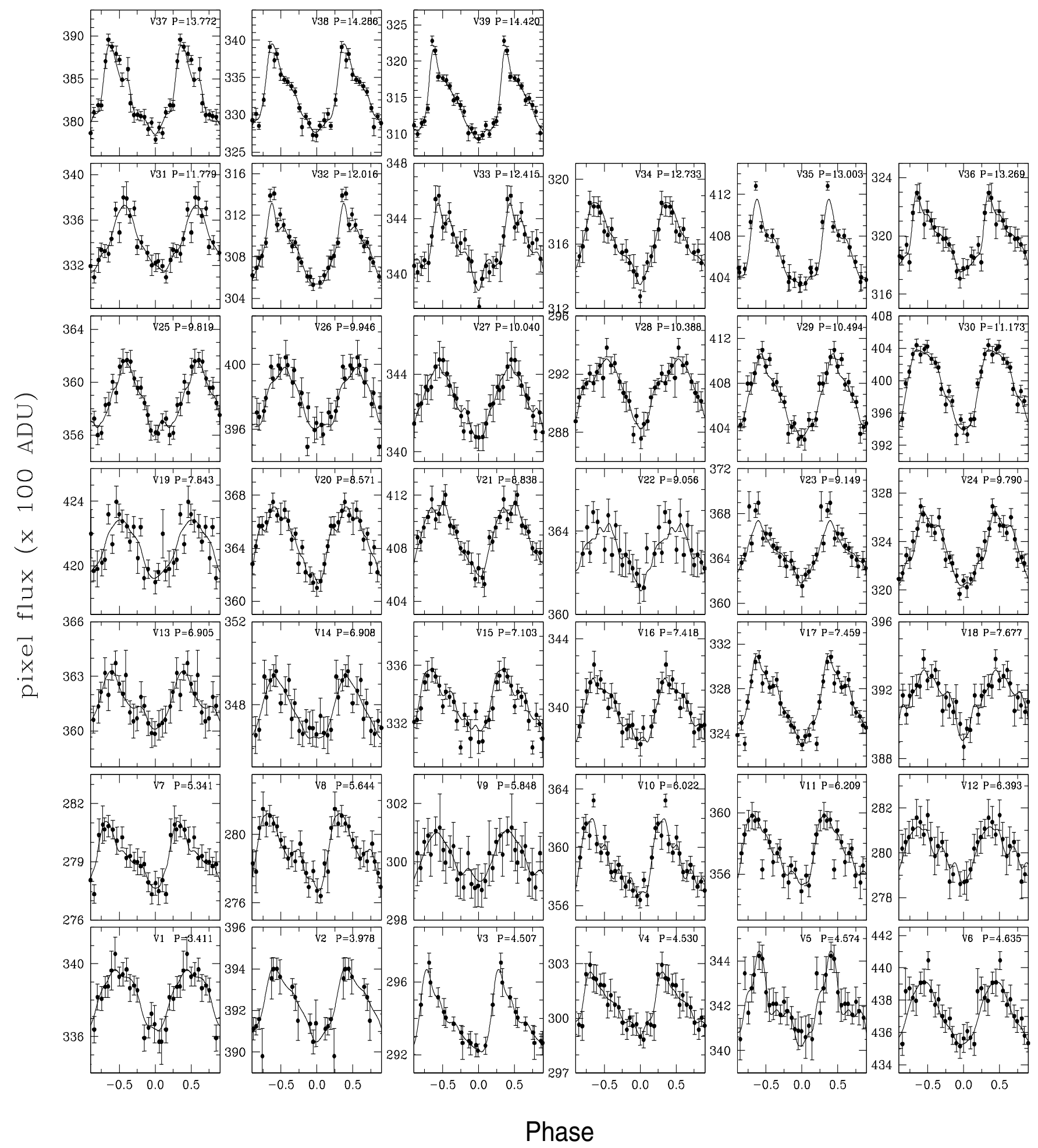

Fig. 1. $R$ band phase light curves of the 39 Cepheids. Periods of the Cepheids are given at the top of each light curve. Phase is plotted twice and in such a way that the minimum flux falls near to zero phase. We use GNUPLOT acsplines routine to interpolate the light curves which approximates the data with a "natural smoothing spline". We have not used statistical errors for the weighting and instead a constant value was used as smoothing weights.

and amplitude of the pulsation in the $R$ band $\left(A_{R}\right)$. The objects are assigned names in the format NMS-M31Vn where $n$ is the Cepheid sequence number and acronym NMS is used for Nainital Microlensing Survey. Celestial coordinates are given for J2000. Whenever any Cepheid is reported as a variable star in the POINT-AGAPE survey catalogue (An et al. 2004, suffixed as PA04), we give their identification number and period in Cols. 8 and 9. If any other references are found corresponding to the Cepheid identified in our study (see the discussion in Sect. 6.1), we give those identifications with prefixes D, J and W respectively for the DIRECT (Kaluzny et al. 1999), Joshi et al. (2003) and WeCAPP (Fliri et al. 2006), in 


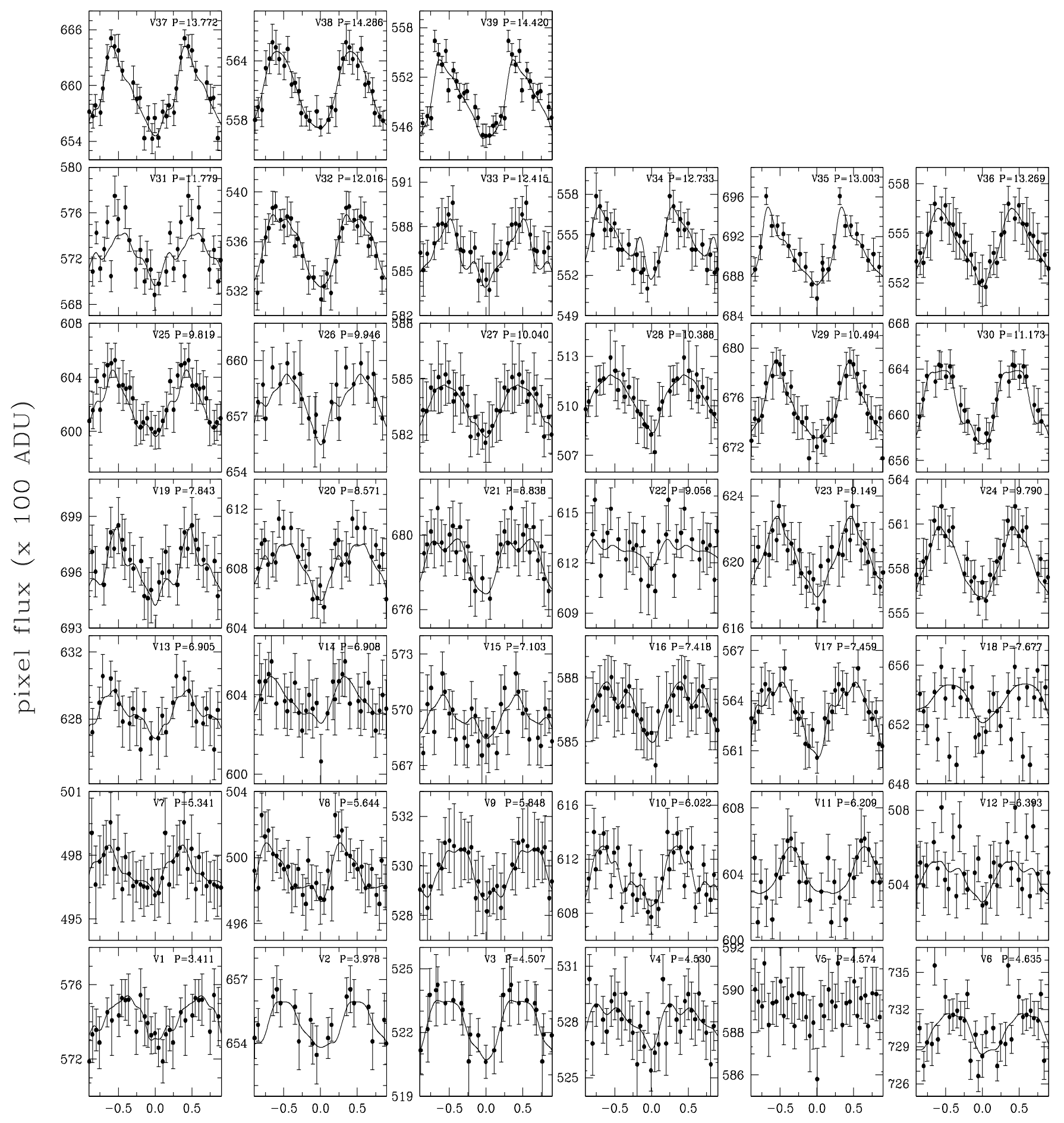

Phase

Fig. 2. Same as in Fig. 1 but for the $I$ band.

the last column of the table. Their corresponding periods are also mentioned in brackets. In the present study, we detected all the 10 Cepheids in the $7-15$ days period range, which were reported in JOS03. However, we identified 15 new Cepheids in the same period range. This was possible thanks mainly to our approach where we search for pixel variability around each pixel instead of identifying stars, and subsequently looking for variability around them after filtering through various selection cuts. On some brighter Cepheids (e.g. M31V38), the smaller amplitude could come from their binary nature or may be significantly blended by nearby bright stars that is unresolved in our observations.

The PSF FWHM of the images used in our analysis varies from 1.5-3.5 arcsec, which is equivalent to about 5 to $12 \mathrm{pc}$ at the distance of $780 \mathrm{kpc}$. This indicates that, despite the large intrinsic brightness of the Cepheids, these stars are most likely to be affected by the flux contribution of hundreds of other neighbouring stars in our target field (i.e., blended), which can significantly 
Table 1. A list of 39 short-period Cepheids identified in the present study with their characteristic parameters.

\begin{tabular}{|c|c|c|c|c|c|c|c|c|c|}
\hline $\begin{array}{l}\text { Cepheid } \\
\text { (NMS-) }\end{array}$ & $\begin{array}{l}\text { RA (J2000) } \\
\text { hh:mm:ss }\end{array}$ & $\begin{array}{c}\operatorname{Dec}(\mathrm{J} 2000) \\
\text { dd:mm:ss }\end{array}$ & $\begin{array}{c}P \\
\text { (Days) }\end{array}$ & $\begin{array}{c}\bar{R} \\
(\mathrm{mag})\end{array}$ & $\begin{array}{c}\bar{I} \\
(\mathrm{mag})\end{array}$ & $\begin{array}{c}A_{R} \\
(\mathrm{mag})\end{array}$ & $\begin{array}{l}\text { PA04 } \\
\text { ID }\end{array}$ & $\begin{array}{c}P \\
\text { (Days) }\end{array}$ & Other identification \\
\hline M31V1 & $00: 43: 48.20$ & $41: 12: 55.9$ & $3.411 \pm 0.001$ & - & - & - & & & \\
\hline M31V2 & $00: 43: 11.14$ & $41: 10: 30.8$ & $3.978 \pm 0.001$ & 22.29 & - & 0.26 & & & \\
\hline M31V3 & 00:44:02.59 & $41: 11: 37.5$ & $4.507 \pm 0.001$ & 21.08 & - & 0.57 & 69301 & 4.508 & \\
\hline M31V4 & 00:43:20.94 & 41:04:07.7 & $4.530 \pm 0.001$ & 20.84 & - & 0.18 & & & \\
\hline M31V5 & $00: 43: 30.24$ & $41: 10: 35.6$ & $4.574 \pm 0.001$ & 21.52 & - & 0.31 & 72533 & 4.634 & \\
\hline M31V6 & $00: 43: 28.65$ & $41: 14: 53.2$ & $4.635 \pm 0.001$ & 20.92 & - & 0.22 & 72356 & 4.581 & \\
\hline M31V7 & $00: 43: 54.95$ & 41:08:12.3 & $5.341 \pm 0.002$ & 21.26 & 20.83 & 0.27 & 69645 & 5.346 & \\
\hline M31V8 & $00: 43: 59.13$ & 41:08:07.8 & $5.644 \pm 0.002$ & 20.78 & 20.06 & 0.22 & 69680 & 5.636 & \\
\hline M31V9 & $00: 43: 19.72$ & $41: 05: 32.4$ & $5.848 \pm 0.004$ & - & - & - & & & \\
\hline M31V10 & $00: 43: 20.88$ & $41: 10: 24.5$ & $6.022 \pm 0.002$ & 21.18 & 20.60 & 0.37 & 74876 & 6.026 & W2583 $(6.021,6.021)$ \\
\hline M31V11 & $00: 43: 21.70$ & 41:08:19.4 & $6.209 \pm 0.003$ & - & - & - & 75216 & 6.209 & \\
\hline M31V12 & $00: 43: 32.84$ & $41: 04: 53.5$ & $6.393 \pm 0.004$ & - & - & - & 73716 & 6.397 & \\
\hline M31V13 & $00: 43: 14.03$ & 41:09:24.9 & $6.905 \pm 0.004$ & 20.67 & 19.70 & 0.13 & & & W1314 $(6.909,6.906)$ \\
\hline M31V14 & $00: 43: 21.06$ & 41:08:14.1 & $6.908 \pm 0.004$ & 20.59 & - & 0.10 & & & W98 $(6.908,6.899)$ \\
\hline M31V15 & $00: 43: 45.28$ & $41: 12: 21.3$ & $7.103 \pm 0.003$ & 20.74 & 20.11 & 0.21 & & & \\
\hline M31V16 & $00: 43: 21.57$ & 41:08:02.4 & $7.418 \pm 0.004$ & 20.74 & - & 0.18 & 75465 & 7.413 & \\
\hline M31V17 & $00: 43: 43.71$ & $41: 11: 48.9$ & $7.459 \pm 0.002$ & 20.55 & - & 0.28 & 71096 & 7.464 & D883 (7.459), J01(7.459) \\
\hline M31V18 & $00: 43: 35.44$ & $41: 15: 05.2$ & $7.677 \pm 0.004$ & 20.41 & 20.34 & 0.13 & & & \\
\hline M31V19 & 00:43:17.49 & $41: 12: 11.3$ & $7.843 \pm 0.004$ & 20.60 & 19.89 & 0.15 & 74607 & 7.852 & W5037 $(7.842,7.849)$ \\
\hline M31V20 & $00: 43: 23.23$ & $41: 10: 25.3$ & $8.571 \pm 0.004$ & 20.16 & 19.67 & 0.17 & 74753 & 8.551 & W2562 (8.567, 8.572), J02(8.566) \\
\hline M31V21 & $00: 43: 28.04$ & $41: 13: 55.4$ & $8.838 \pm 0.003$ & 20.71 & 20.46 & 0.27 & 72015 & 8.831 & $\mathrm{~J} 03(8.836)$ \\
\hline M31V22 & $00: 43: 45.37$ & $41: 15: 09.7$ & $9.056 \pm 0.012$ & - & - & - & & & \\
\hline M31V23 & $00: 43: 44.82$ & $41: 15: 01.0$ & $9.149 \pm 0.009$ & 20.37 & 19.65 & 0.20 & & & D1219 (9.173), J04(9.160) \\
\hline M31V24 & $00: 43: 53.27$ & $41: 12: 46.1$ & $9.790 \pm 0.004$ & 20.58 & 20.10 & 0.25 & 70319 & 9.772 & D2879 (9.790), J05(9.790) \\
\hline M31V25 & $00: 43: 33.65$ & $41: 11: 52.9$ & $9.819 \pm 0.005$ & 20.76 & - & 0.26 & 72649 & 9.550 & \\
\hline M31V26 & $00: 43: 38.79$ & $41: 15: 53.8$ & $9.946 \pm 0.009$ & 20.14 & 19.83 & 0.11 & & & \\
\hline M31V27 & $00: 43: 40.67$ & $41: 12: 44.8$ & $10.040 \pm 0.004$ & 20.93 & 20.64 & 0.23 & 69993 & 10.023 & \\
\hline M31V28 & 00:43:30.49 & $41: 03: 36.4$ & $10.388 \pm 0.006$ & 20.53 & 20.27 & 0.20 & 87421 & 10.375 & J06(10.383) \\
\hline M31V29 & $00: 43: 29.67$ & $41: 14: 12.0$ & $10.494 \pm 0.004$ & 20.71 & 20.35 & 0.34 & 72289 & 10.495 & $\mathrm{~J} 07(10.500)$ \\
\hline M31V30 & 00:43:00.01 & 41:08:33.3 & $11.173 \pm 0.006$ & 20.04 & 20.25 & 0.27 & & & W490 (11.168, 11.172), J08(11.19) \\
\hline M31V31 & $00: 43: 51.25$ & $41: 14: 24.0$ & $11.779 \pm 0.013$ & 20.95 & 20.26 & 0.34 & & & \\
\hline M31V32 & $00: 43: 21.70$ & $41: 05: 02.4$ & $12.016 \pm 0.009$ & 20.49 & 20.06 & 0.29 & 75721 & 12.050 & \\
\hline M31V33 & $00: 43: 28.98$ & $41: 10: 12.8$ & $12.415 \pm 0.008$ & - & - & - & 72505 & 12.417 & \\
\hline M31V34 & 00:43:50.98 & $41: 12: 56.9$ & $12.733 \pm 0.005$ & 21.81 & 20.85 & 0.74 & 70598 & 12.706 & \\
\hline M31V35 & 00:43:35.09 & $41: 15: 32.0$ & $13.003 \pm 0.007$ & 20.74 & 19.56 & 0.36 & & & \\
\hline M31V36 & $00: 43: 46.67$ & $41: 11: 30.0$ & $13.272 \pm 0.007$ & 20.60 & 20.14 & 0.21 & 71271 & 13.274 & \\
\hline M31V37 & $00: 43: 26.25$ & $41: 12: 01.6$ & $13.770 \pm 0.006$ & 20.26 & 19.68 & 0.31 & 72459 & 13.772 & J09(13.773) \\
\hline M31V38 & $00: 43: 47.94$ & 41:10:02.6 & $14.286 \pm 0.006$ & 19.53 & 19.09 & 0.16 & 71168 & 14.256 & \\
\hline M31V39 & $00: 43: 42.97$ & $41: 10: 17.6$ & $14.420 \pm 0.005$ & 20.86 & 19.97 & 0.56 & 70712 & 14.454 & $\mathrm{~J} 10(14.420)$ \\
\hline
\end{tabular}

increase their observed magnitude and decrease the amplitude. Using high-resolution HST images, Mochejska et al. (2000) concludes that the average flux contribution from the bright companions that are not resolved on the ground-based images is about $19 \%$ of the flux of the Cepheid in $V$ band. Macri et al. (2006) points out that fainter and low-amplitude Cepheids like those detected in our pixel survey are more affected by this blending problem. In general, different estimates put a blending of 0.1 to 0.3 mag in $B$ and $V$ bands in M31 Cepheids (Mochejska et al. 2000; Kiss \& Bedding 2005; Vilardell et al. 2007, and references therein). In the present case, it could be even larger due to choice of our filters ( $R$ and $I$ bands) since large numbers of red stars are present in M31. We therefore caution readers that the magnitudes and amplitude for the Cepheids given in Table 1 should not be considered definitive, and much more precise photometry is needed to accurately determine these values.

One major problem of our analysis is the uncertainty in determining of the colour $(R-I)$, which is, unfortunately, mainly caused by the poor light curves and smaller phase sampling in the $I$ band, as well as the blending problem. Therefore, we are not in a position to discuss the colour-magnitude diagram of these Cepheids.

\section{Discussion}

\subsection{Comparison with other catalogues}

The list of Cepheids reported here is compared with those surveys having identified variable stars within our relatively small field of view. A comparison with the $V$-band photometric observations of Magnier et al. (1997) and BVI photometric observations carried out by the DIRECT project (Kaluzny et al. 1999) has already been done in JOS03. No additional shortperiod Cepheids in common have been found, since all of them have already been identified previously.

The WeCAPP microlensing survey recently reported a catalogue of 23781 variable stars including 33 population I Cepheids 


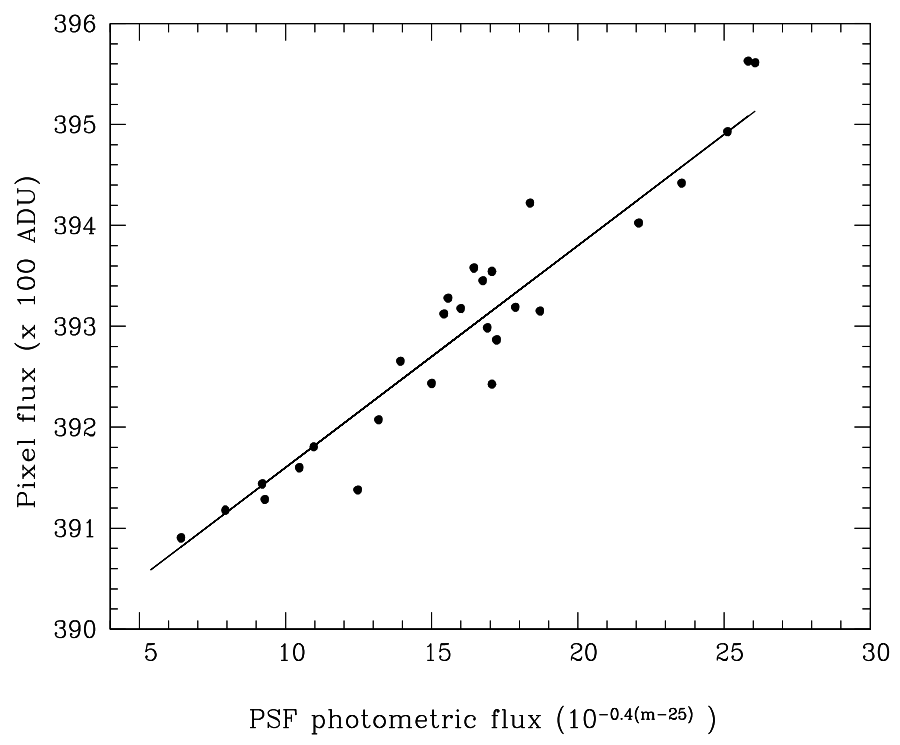

Fig. 3. A correlation between photometric fluxes determined from PSFfitted photometric magnitudes and corresponding pixel fluxes of a Cepheid NMS-M31V2 identified in our survey.

and 93 population II Cepheids (Fliri et al. 2006). Although the WeCAPP survey mostly observed towards the bulge of M31, only a small portion of our target field was common with them, where they found 11 Population I Cepheids. However, we identified only 6 of them in our survey, because the rest of them were too faint to be detected in our photometry. For each Cepheid, WeCAPP survey has given two different periods in $R$ and $I$ bands and we found our periods agree to within 0.001 day with one of their two periods given for any Cepheid (see, Col. 10 of Table 1).

The most complete list of variable stars in M31 to date is given by the POINT-AGAPE microlensing survey, which has produced an exhaustive list of 35414 variable stars in M 31 (An et al. 2004). It is worth mentioning here that the POINT-AGAPE survey did not characterize their variable stars as Cepheids and just listed them as the variables in their catalogue. Two of their fields ( 7 and 8) fall in our target field. We found 25 Cepheids in the present study that were also listed in their catalogue. The celestial coordinate of some of the common variables between two surveys are separated by as much as 4 arcsec, but found with almost the same period. This could represent the typical combined astrometric inaccuracy between these two catalogues. Furthermore, some of their variable stars were found less than 5 arcsec away from each other. For example, one of our Cepheid NMS-M31V8 matches two of their variable stars with ID numbers 69654 and 69680 which lie at a separation of just 2 arcesc but identified with vastly different period of 171.791 and 5.636 days, respectively, in their survey. In our analysis, we determined a period of 5.644 days for this Cepheid, which is close to the later period. We therefore considered only those Cepheids that correspond to our identified Cepheids where two periods match within a day in two surveys. It is possible for POINTAGAPE to have two variables detected in such close proximity with a completely different periods, due to their relatively deeper photometry and better sky conditions in La Palma. In Cols. 8 and 9 of Table 1, we list the ID and period of the stars found in POINT-AGAPE survey, which closely matches with the Cepheids found in the present study. Most of the periods agree within 0.06 days of each other, except NMS-M31V25, which has a period difference of 0.269 days.

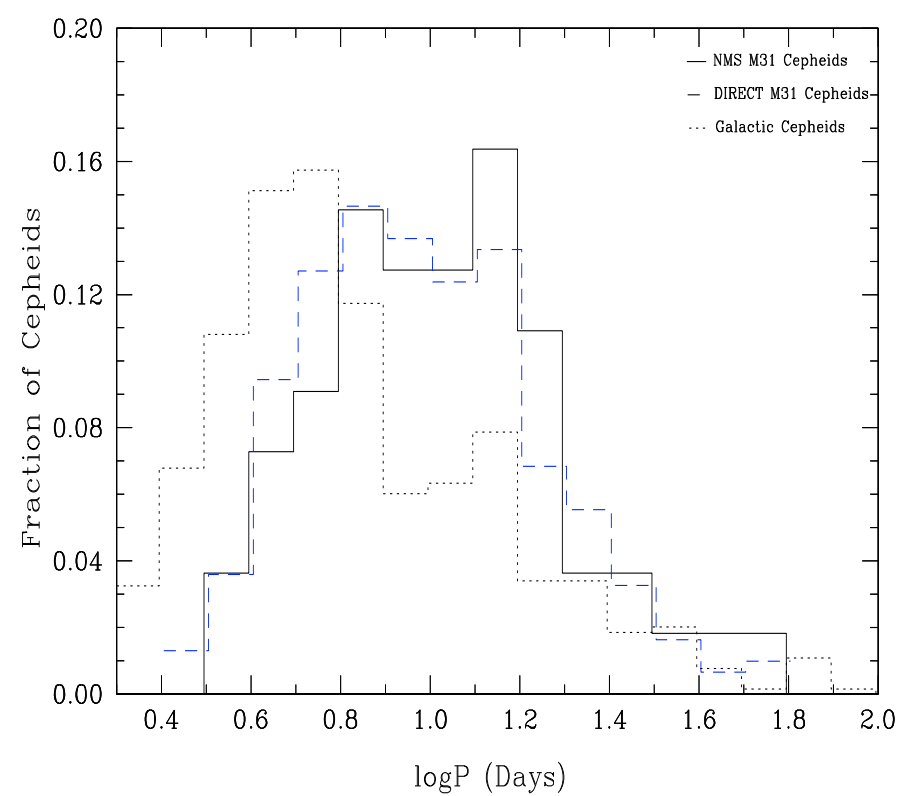

Fig. 4. The normalized frequency-period distribution of Cepheids as a function of their pulsation period in logarithmic scale. Cepheids identified in our target field detected under Nainital Microlensing Survey and DIRECT survey are shown by solid and dashed lines while Milky Way Cepheids taken from the GCVS catalogue are plotted by the dotted line. The adopted bin size in all the 3 distributions is $0.1 \mathrm{in} \log P$.

\subsection{Frequency-period distribution of the Cepheids}

The number of Cepheids observed in a galaxy is not uniformly distributed over all possible periods. The number of Cepheids occurring over a certain range of periods in a given complete sample depends on the initial mass function, chemical composition of the galaxy, and the structure and evolutionary time scales of stars of different masses during their transit through the instability strip. The frequency-period distribution for classical Cepheids has been studied in detail by Becker et al. (1977), Serrano (1983), and Alcock et al. (1999) for different galaxies and their studies show that the frequency-period distribution is a function of chemical composition. Serrano (1983) also pointed out that the mean period of Cepheids decreases with the galactocentric distance. Two different explanations have been given to explain the bimodal pattern in frequency-period distribution of Cepheids and deficiency of 8-10 days period by Becker et al. (1977) and Boucher et al. (1997). While the former noted that it is two-component birth-rate function responsible for the double peak, later suggested that it is in fact nonlinear fundamental pulsation cycle in 8-10 days range where corresponding Cepheids pulsate in the first overtone having period $P_{1} \approx 0.7 P_{0}$, resulting in an overall increase in overtone Cepheids in the period range 5.6-7.0 days period, which in turn shows a double peak in the frequency-period distribution. Recently Antonello et al. (2002) has done an extensive study of the frequency-period distribution of 6 local group of galaxies and demonstrated that, while 3 metal-poor galaxies, i.e. LMC, SMC and IC 1613, do not show any conspicuous bimodal distribution in the frequency-period diagram, other 3 metal-rich galaxies i.e. Milky Way, M31, and M 33 have a visibly seen bimodal distribution.

To understand the period distribution of Cepheids in the present study, we compared it with the DIRECT survey (Macri 2004, and references theirin) where we found 332 Cepheids in six of their targeted M 31 field, and 25 of them were repetitions among different fields. We determined the fractional distribution 
of Cepheids against $\log P$ for the two catalogues-our catalogue of 55 Cepheids detected as a whole in the Nainital Microlensing Survey and 307 Cepheids detected in the DIRECT survey. We estimated the frequency of Cepheids in each 0.1 bin of $\log P$ in both cases and normalized them with the total number of Cepheids detected in the respective catalogues. In Fig. 4, we plot histograms of these distributions. Although our conclusion is based on a fairly small sample of Cepheids observed in a small region of M 31 disk in our survey and subject to statistical error, but it clearly shows that period distribution of Cepheids observed in M31 shows a bimodal pattern with peaks around $\log P \sim$ 0.9 and 1.1 days, a similar pattern as shown by the DIRECT Cepheids. However, Antonello et al. (2002) find primary and secondary maxima at $\log P \sim 0.7$ and 1.1 , respectively using the sample of M31 Cepheids reported in the GCVS catalogue while Vilardell et al. (2007) find these peaks close to 0.6 and 1.1 in their survey. On comparing the M 31 Cepheids frequencyperiod distribution with that of the Milky Way, we observed a bimodal period distribution using 648 Galactic Cepheids reported in the GCVS catalogue with peaks around $\log P \sim 0.7$ and 1.1 days (see, Fig. 4), a pattern also seen by Vilardell et al. (2007) who used the David Dunlop Observatory catalogue of Galactic Cepheids (Fernie et al. 1995).

It is quite evident that the frequency-period distribution in M 31 vary in shape and in the location of the peak among different surveys. This is possibly caused by the incompleteness of the Cepheids detected within these surveys. While GCVS and DIRECT surveys seem incomplete towards a shorter period, Vilardell et al. (2007) has attributed it to the observational biases for the long-period Cepheids in their data. Apart from the limiting magnitude of each surveys, these surveys are biased in their detection of Cepheids because of the non-uniform sampling of the data in different regions of the M 31, non-detection of lowamplitude Cepheids and blending of Cepheids by either foreground stars or nearby bright stars within the host galaxy itself. To better understand the period distributions of the Cepheids in M 31, a systematic and deep photometric search of the galaxy is needed to obtain a homogeneous data sample, at least in $B V I$ bands.

\subsection{Period-luminosity relation}

The Cepheid variables exhibit an excellent correlation between their mean intrinsic brightness and pulsation period and widely used as standard candles for estimating extragalactic distances by comparing their absolute magnitudes inferred from the period-luminosity relation with their observed apparent magnitudes. However, as discussed earlier, short-period and lowamplitude Cepheids are expected to be affected by blending, so the error in their mean magnitude is possibly dominated by this as opposed to the photometric error itself. JOS03 reported detection of 26 Cepheids and 10 of these are detected in this study. A period-luminosity diagram of the Cepheids detected in our survey is shown in Fig. 5. Here, we used all 55 Cepheids detected in our survey, except for the 6 Cepheids for which we could not determine their $R$ magnitude. We note that M 31 Cepheids identified in our target field could have more than $30 \%$ inaccuracy in their reported magnitudes because of the combined effect of photometric error, errors in flux correlation, and blending effect, etc. Furthermore, while Vilardell et al. (2007) suggest not using Cepheids below 0.8 mag amplitude in $V$ band in deriving the precise distance of M31, we chose not to implement any such criteria in the present study owing to the large number of low amplitude Cepheids detected in our survey.

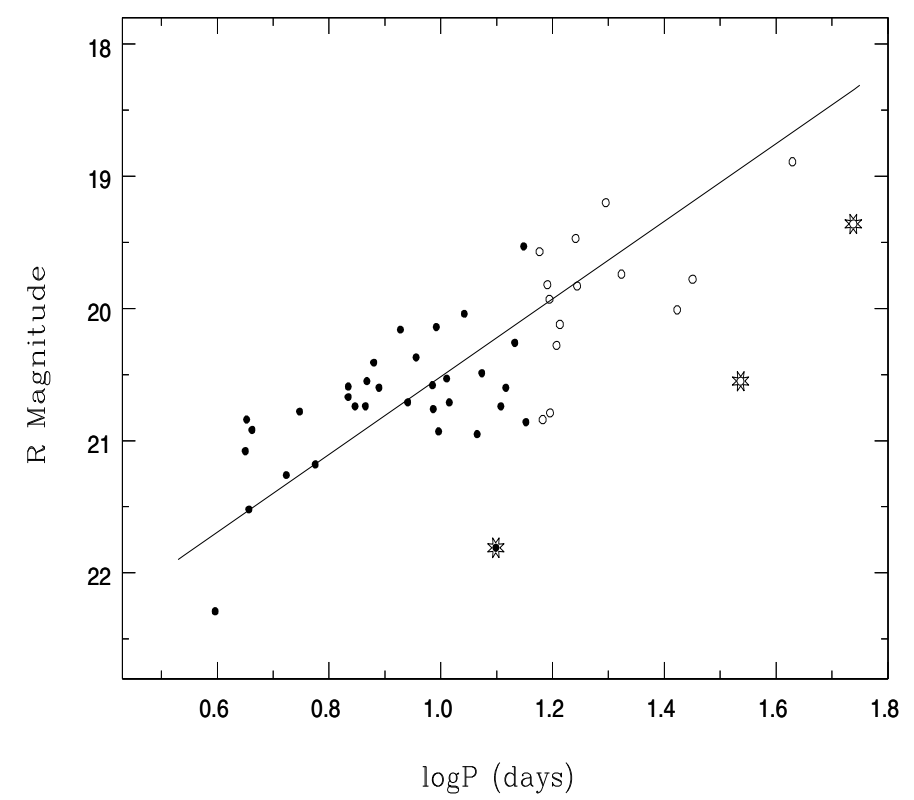

Fig. 5. $R$ band Period-luminosity diagram for 49 Cepheids for which we have mean $R$ magnitude available (see Table 1). Filled circles indicate short-period Cepheids identified in the present study and open circles indicate the Cepheids with $P>15$ days identified in the previous study. The slope of the fitted straight lines are fixed at $\mathrm{dm} / \mathrm{d} \log P=-2.94$, given by Madore \& Freedman (1991). 3 Cepehids marked with asterisk could be possible population II Cepheids.

To estimate the distance of M 31 from the $R$ band periodluminosity diagram, we kept the slope and zero point fixed at -2.94 and -4.52 , respectively (Madore \& Freedman 1991), and used a total extinction of 0.63 in our observed direction (JOS03). We excluded 3 Cepheids from our sample for the distance estimation, which could be Population II objects as they fall about 1.5 to 2 mag below the period-luminosity relation for the classical Cepheids (indicated by an asterisk in Fig. 5). We determined a distance modulus of $(m-M)_{0}=24.41 \pm 0.21 \pm 0.30 \mathrm{mag}$ for M31. Here the first error indicates uncertainty in the zero point, while second error indicates typical photometric error at the faintest magnitude level in our $R$ band data. Though our distance estimate is consistent with those previously found for M 31 (e.g. Freedman et al. 2001; Brown et al. 2004; Vilardell et al. 2007), we emphasize that the distance estimation based on the M31 Cepheids identified in our survey is a crude estimation, so much more precise photometry of these Cepheids at multiple wavelengths is needed to ascertain an accurate distance to M 31 .

\section{Summary}

The main aim of the "Nainital Microlensing Survey" was to search for microlensing events in the direction of M31. However, the vast amount of data also enabled us to identify a substantial number of variable stars and optical transients in the $13^{\prime} \times 13^{\prime}$ region of the M31 disk. The data in the present study was analysed using the pixel technique that is commonly used to look variability in the crowded fields and/or poor seeing conditions. In this study, we present a catalogue of 39 shortperiod Cepheids in the disk of M31, which were found within the magnitude range $\sim 19.5-22.3$ in $R$ band. Several photometric observations carried out over the survey's 4 years duration have allowed us to determine their periods, which was found in the range between $\sim 3$ to 15 days. Although the phase coverage of the 
Cepheids is uneven in our survey, these light curves cover many cycles of periodic variations, so that derived periods are reliable and generally in good agreement with previously published values. A correlation between PSF-fitted photometric magnitudes, whenever possible, and corresponding pixel fluxes were used to calculate mean magnitude and amplitude of variability of these Cepheids in $R$ and $I$ bands. Fourier analysis is often used to distinguish classical Cepheids from those pulsating in their firstovertone (Vilardell et al. 2007, and references theirin); however, the photometric quality of our data is not good enough to study the pulsation modes of these Cepheids.

It is quite evident that, while the long-period Cepheids are well represented in most of the surveys, short-period Cepheids are under-sampled due to their low-amplitude, low intrinsic brightness, and poor data sampling. It is also difficult to detect short-period Cepheids in a crowded field like M31 where the faint stars, particularly in poor seeing conditions, are visually undetectable from the background flux. However, it was demonstrated that a much more complete sample of Cepheids can be obtained, even among short-period and intrinsically faint Cepheids, using the pixel technique. Our observation of bimodal frequency-period distribution in a sample of 55 M 31 Cepheids detected in the Nainital Microlensing Survey agrees with such a trend seen by the other surveys; however, a systematic search for the Cepheids is required to fully understand the underlying reasons for the variations in shape and location of the peak in the frequency-period distributions among different surveys. Owing to observing limitations, the present sample does not contain stars with $P$ shorter than 3.4 days, and the large uncertainties in our magnitudes did not allow us to compare our results with the other galaxies on the basis of their metallicities.

The growing number of Cepheids in distant galaxies are not only useful for determining their precise distances but also for tracing star formation history of the galaxy itself. Although our field of view is smaller than some other wide-field surveys carried out in M 31, our catalogue of short-period Cepheids detected through pixel method bring a significant contribution towards lower branch of the period-luminosity diagram, and our results show that, despite average quality data, we can get comparative results with the other surveys.

Acknowledgements. It is a great pleasure to thank Yannick Giraud-Héraud and Jean Kaplan for their kind support in initiating this project and helping us to become familiar with the pixel technique. We are thankful to Don Pollacco for his useful comments on the initial draft of this paper and to the supporting staff at the Nainital observatory for their help in carrying out sucessful observations for such a long period. This study is a part of the project 2404-3 supported by the Indo-French centre for the Promotion of Advanced Research, New Delhi.

\section{References}

Alcock, C., Allsman, R. A., Alves, D. R., et al. 1999, AJ, 117, 920 An, J. H., Evans, N. W., Hewett, P., et al. 2004, MNRAS, 351, 1071 Ansari, R., Aurière, M., Baillon, P., et al. 1997, A\&A, 324, 843 Ansari, R., Aurière, M., Baillon, P., et al. 1999, A\&A, 344, L49 Ansari, R., Aurière, M., Baillon, P., et al. 2004, A\&A, 421, 509 Antonello, E., Fugazza, D., \& Mantegazza, L. 2002, A\&A, 388, 477 Auriére, M., Baillon, P., Bouquet, A., et al. 2001, ApJ, 553, L137 Baillon, P., Bouquet, A., Giraud-Héraud, Y., \& Kaplan, J. 1993, A\&A, 277,1 Becker, S. A., Iben, I. Jr., \& Tuggle, R. S. 1977, ApJ, 218, 633 Brown, T. M., Ferguson, H. C., Smith, E., et al. 2004, AJ, 127, 2738 Buchler, J. R., Goupil, M. J., \& Piciullo, R. 1997, ApJ, 491, L99 Crotts, A. P. S., \& Tomaney, A. B. 1996, ApJ, 473, L87 Darnley, M. J., Bode, M. F., Kerins, E., et al. 2004, MNRAS, 353, 571 de Jong, J. T. A., Kuijken, K., Crotts, A. P. S., et al. 2004, A\&A, 417, 461 Fernie, J. D., Evans, N. R., Beattie, B., \& Seager, S. 1995, Infor. Bull. Variable Stars, 4148, 1

Fliri, J., Riffeser, A., Seitz, S., \& Bender, R. 2006, A\&A, 445, 423

Freedman, W. L, Madore, B. F., Gibson, B. K., et al. 2001, ApJ, 553, 47 Horne, J. H., \& Baliunas S. L. 1986, ApJ, 302, 757

Joshi, Y. C., Pandey, A. K., Narasimha, D., Sagar, R., \& Giraud-Héraud, Y. 2003, A\&A, 402, 113 (JOS03)

Joshi, Y. C., Pandey, A. K., Narasimha, D., et al. 2004, A\&A, 415, 471

Joshi, Y. C., Pandey, A. K., Narasimha, D., \& Sagar, R. 2005, A\&A, 433, 787 113

Kaluzny, J., Mochejska, B. J., Stanek, K. Z., et al. 1999, AJ, 118, 346

Kerins, E., Darnley, M. J., Duke, J. P., et al. 2006, MNRAS, 365, 1099

Kiss, L. L., \& Bedding, T. R. 2005, MNRAS, 358, 883

Landolt, A. U. 1992, AJ, 104, 340

Macri, L. M. 2004, Variable Stars in the Local Group, ed. D. W. Kurtz, \& K. Pollard, ASP Conf. Ser., 310, 33

Macri, L. M., Stanek, K. Z., Bersier, D., Greenhill, L. J., \& Reid, M. J. 2006, ApJ, 652, 1133

Madore, B. F., \& Freedman, W. L. 1991, PASP, 103, 933

Magnier, E. A., Lewin, W. H. G., van Paradijs, J., et al. 1992, A\&AS, 96, 379

Magnier, E. A., Augusteijn T., Prins, S., van Paradijs, J., \& Lewin, W. H. G. 1997, A\&AS, 126, 401

Mochejska, B. J., Macri, L. M., Sasselov, D. D., \& Stanek, K. Z. 2000, AJ, 120, 810

Monet, D. G., Levine, S. E., Canzian, B., et al. 2003, AJ, 125, 984

Paulin-Henriksson, S., Baillon, P., Bouquet, A., et al. 2003, A\&A, 405, 15

Press, W. H., \& Rybici G. B. 1989, ApJ, 338, 277

Riffeser, A., Fliri, J., \& Gössl, C. A. 2001, A\&A, 379, 362

Serrano, A. 1983, RMxAA, 8, 131

Stetson, P. B. 1987, PASP, 99, 191

Vilardell, F., Jordi, C., \& Ribas, I. 2007, A\&A, 473, 847 